\title{
Model for End-Stage Liver Disease and Sodium Velocity Predicts Overall Survival in Nonmetastatic Hepatocellular Carcinoma Patients
}

\author{
Justin Y. Tang ${ }^{\mathbb{D},}{ }^{1}$ Nitin Ohri, ${ }^{1}$ Rafi Kabarriti, ${ }^{1}$ Santiago Aparo, ${ }^{2}$ \\ Jennifer Chuy, ${ }^{2}$ Sanjay Goel, ${ }^{2}$ Jonathan M. Schwartz, ${ }^{3}$ Milan Kinkhabwala, ${ }^{4}$ \\ Andreas Kaubisch ${ }^{D}{ }^{2}$ and Chandan Guha ${ }^{1}{ }^{1}$ \\ ${ }^{1}$ Department of Radiation Oncology, Montefiore Medical Center and Albert Einstein College of Medicine, Bronx, NY, USA \\ ${ }^{2}$ Department of Medicine, Division of Medical Oncology, Montefiore Medical Center and Albert Einstein College of Medicine, \\ Bronx, NY, USA \\ ${ }^{3}$ Department of Gastroenterology and Liver Diseases, Montefiore Medical Center and Albert Einstein College of Medicine, \\ Bronx, NY, USA \\ ${ }^{4}$ Department of Surgery, Montefiore Medical Center and Albert Einstein College of Medicine, Bronx, NY, USA
}

Correspondence should be addressed to Chandan Guha; cguha@montefiore.org

Received 21 May 2018; Revised 7 October 2018; Accepted 21 October 2018; Published 7 November 2018

Academic Editor: Pascal Lapierre

Copyright (C) 2018 Justin Y. Tang et al. This is an open access article distributed under the Creative Commons Attribution License, which permits unrestricted use, distribution, and reproduction in any medium, provided the original work is properly cited.

Background \& Aims. The significance of short-term changes in model for end-stage liver disease and Sodium (MELD-Na) following hepatocellular carcinoma (HCC) diagnosis is unknown. In this report, we explore the value of the rate of short-term changes in MELD-Na as an independent predictor of mortality in patients with nonmetastatic HCC. Methods. We reviewed a cohort of patients diagnosed with nonmetastatic HCC at our institution between 2001 and 2011. We evaluated potential predictors of overall survival, including baseline MELD-Na and the change in MELD-Na over 90 days. We explored survival times of cohorts grouped by baseline MELD-Na and the change in MELD-Na. Results. 182 patients met eligibility criteria. With a median follow-up of 21 months for surviving patients, 110 deaths were observed (60\%). Median MELD-Na at the time of diagnosis was 9.7 (IQR 7.5 to 13.9). The median changes in percentage of MELD-Na over 90 days were an increase of 9\% (IQR - 4\% to 55\%). Multivariable Cox proportional hazards modeling demonstrated that both baseline MELD-Na (HR=1.07 per unit increase, $95 \%$ CI 1.03 to $1.11, p<0.001)$ and changes in MELD-Na exceeding 40\% ( $\mathrm{HR}=3.69,95 \% \mathrm{CI} 2.39$ to 5.69, $\mathrm{p}<0.001)$ were independently associated with increased mortality risk. Median survival among patients whose changes in MELD-Na were greater than $40 \%$ was 4.5 months, and median survival among the 131 other patients was 25.8 months $(\mathrm{p}<0.001)$. Conclusions. We identified a subset of HCC patients who have extremely poor prognosis by incorporating the rate of short-term change in MELD-Na to baseline MELD-Na score.

\section{Introduction}

In 2013, the American Cancer Society estimated that over 30,000 new cases of hepatocellular carcinoma (HCC) were diagnosed annually in the United States. The incidence of HCC in the United States is increasing [1-3]. Individuals with HCC in the United States have a 5-year survival rate of only 8.9\% despite aggressive treatment [2]. Liver transplantation and local resection remain the preferred curative treatment options for early stage disease $[3,4]$.
The model for end-stage liver disease (MELD) is a scoring system used to predict three-month mortality in patients with advanced liver disease. It is calculated from three biochemical variables that are markers for direct and indirect measures of hepatic functions: creatinine, prothrombin time (INR), and serum bilirubin. Rising MELD scores indicate deterioration in liver function $[5,6]$. The MELD (and PELD for pediatrics) scoring system was incorporated into liver transplant allocation in order to stratify patients on waiting list according to the severity of illness [7]. More recently, 
MELD was explored as a means of predicting prognosis in HCC patients $[8,9]$, building on the previous work of investigators who incorporated severity of liver disease in cancer staging (Barcelona). Furthermore, the predictive value of MELD was improved with the addition of serum sodium in the equation (MELD-Na), based on the observation that dilutional hyponatremia is a poor prognostic sign in advanced cirrhosis and portal hypertension $[10,11]$. This was established in a cohort of HCC patients who were predominantly Asian and infected with hepatitis B (HBV) [11].

Our cancer center population differs from the original study population in which MELD-Na was established with respect to race, socioeconomic factors, and HCC etiologies. While HBV infection is more prevalent worldwide, hepatitis $\mathrm{C}(\mathrm{HCV})$ is more prevalent in the United States $[1,3]$. Recent studies have demonstrated differences in clinical features and prognosis between HBV and HCV-induced HCC [1214]. The applicability of MELD-Na in the setting of HCVinduced HCC warrants further evaluation. In addition, we hypothesized that the velocity of short-term changes in MELD-Na will give additional prognostic information in this setting.

The aim of this study was to analyze MELD-Na and changes in MELD-Na as prognostic markers of survival in patients with nonmetastatic HCC using a retrospective cohort from a single large US center.

\section{Patients and Methods}

2.1. Patient Selection. This study was approved by the Albert Einstein College of Medicine Institutional Review Board (IRB). The IRB waived the informed consent process as the study was retrospective and patient identifiers were deidentified. We utilized the Montefiore Medical Center (Bronx, NY) cancer registry to identify patients diagnosed with HCC between 2001 and 2011. Patients with a diagnosis of hepatocellular carcinoma with sufficient data to calculate MELD-Na at the time of diagnosis (within 30 days) and approximately 90 days after diagnosis (MELD-Na_90) were included in the study. Patient characteristics were tabulated using Clinical Looking Glass (CLG); an electronic medical record research tool developed at our institution [15]. Date of death was collected using social security death registry, and data was censored at the end of 2011, which is the last date that the full social security death registry is available. Patients who underwent liver transplantation were excluded, as were patients with extrahepatic (Stage IV) disease. The patient data used to support the findings of this study are restricted by the Albert Einstein College of Medicine Institutional Review Board in order to protect patient privacy. Data are available for researchers who meet the criteria for access to confidential data.

2.2. Measures. Abstracted data included age at diagnosis, gender, race (categorized as White, Black, Asian, and others), ethnicity (Hispanic and non-Hispanic), HCV infection (defined as either having ICD9 diagnosis, positive HCV antibody by enzyme immunoassay or detectable HCV RNA by PCR), HBV infection (defined as either having ICD9 diagnosis, positive $\mathrm{HBV}$ core antigen, or detectable $\mathrm{HBV}$ DNA by polymerase chain reaction), alcohol use (defined as ICD9 documentation of alcohol abuse or documentation of alcohol use), alpha fetoprotein (AFP) level at diagnosis (defined as AFP level drawn within 30 days of HCC diagnosis), tumor stage (defined by American Joint Committee on Cancer Criteria version 6.0), and treatment modalities (systemic treatment, hepatectomy, transplantation, TACE, or RFA). Bilirubin, creatinine, INR, and sodium values from within 30 days of HCC diagnosis and 90 days after HCC diagnosis were tabulated and used to calculate MELD-Na and MELD-Na_90, respectively.

Each MELD score was calculated using the formula: $3.78 * \ln ($ bilirubin $)+11.2 * \ln ($ INR $)+9.57 * \ln ($ creatinine $)+$ 6.43. The minimal value of bilirubin, INR, and creatinine was changed to 1 in the score calculation according to convention. Likewise, the maximal value of creatinine was capped at 4 . MELD-Na score was calculated using the formula: MELD $+1.59 *(135$-sodium $)$ with serum sodium minimum and maximum range of $120-135 \mathrm{mEq} / \mathrm{L}$ [11]. The velocity of shortterm changes in MELD-Na was calculated as the percent change from baseline MELD-Na scores to MELD-Na_90 scores.

2.3. Statistical Analyses. Descriptive statistics were used to report patient characteristics. AFP at the time of diagnosis was dichotomized at $400 \mathrm{ng} / \mathrm{ml}$, which has been demonstrated to be an important prognostic cutoff in the past [16].

Univariate Cox proportional hazards models were built to identify associations between baseline clinical variables and overall survival. A multivariable model was built utilizing a backwards stepwise approach. Kaplan-Meier curves for overall survival were generated after dividing patients into groups of equal sizes based on baseline MELD-Na. Statistical comparisons were performed using log-rank testing.

We tested cut-points for the changes in MELD-Na increase ranging from $0 \%$ to $100 \%$ (in 5\% intervals) as predictors of mortality in univariate Cox proportional hazards models. The cut-point that yielded the lowest $\mathrm{p}$ value, with the stipulation that at least $25 \%$ of patients fell above and below the cut-point, was selected as the optimal value. This binary variable was tested in univariate and multivariable survival models that included clinical characteristics and baseline MELD-Na. Additional variables were added to the final multivariable model to test for interactions between statistically significant independent variables as predictors of survival. Kaplan-Meier curves for overall survival were generated after dividing patients into two groups based on the optimal cutoff for MELD-Na increase. Statistical comparisons were performed using log-rank testing. Kaplan-Meier curves were also generated after dividing patients into four groups based on baseline MELD-N (below/above median) and MELD-Na increase (below/above optimal cutoff).

Statistical analyses were performed using STATA 12.1 (StataCorp, College Station, TX) and Matlab 8.4 (The Mathworks, Natick, MA). All reported p values were two-sided, and a $\mathrm{p}$ value cutoff of 0.05 was used to determine statistical significance. Survival times were calculated from the date of diagnosis. 
TABLE 1: Characteristics of the 182 subjects included in this study.

\begin{tabular}{|c|c|}
\hline Characteristic & $\mathrm{N}=182$ \\
\hline Age, mean (SD) & $61.8(11.1)$ \\
\hline \multicolumn{2}{|l|}{ Gender, n (\%) } \\
\hline Male & $133(73 \%)$ \\
\hline Female & $49(27 \%)$ \\
\hline \multicolumn{2}{|l|}{ Stage, n (\%) } \\
\hline I & $65(36 \%)$ \\
\hline II & $51(66 \%)$ \\
\hline III & $66(36)$ \\
\hline \multicolumn{2}{|l|}{$\operatorname{AFP}(\mathrm{ng} / \mathrm{mL})$} \\
\hline$<400$ & $127(70 \%)$ \\
\hline$>400$ & $55(30 \%)$ \\
\hline \multicolumn{2}{|l|}{ Race, n (\%) } \\
\hline White & 107 (59\%) \\
\hline Black/African American & $54(30 \%)$ \\
\hline Asian & $5(2 \%)$ \\
\hline Other/Unknown & $16(9 \%)$ \\
\hline \multicolumn{2}{|l|}{ Ethnicity, n (\%) } \\
\hline Not Hispanic/Latino & $85(47 \%)$ \\
\hline Hispanic/Latino & $97(54 \%)$ \\
\hline \multicolumn{2}{|l|}{ Etiology of cirrhosis, n (\%) } \\
\hline $\mathrm{HCV}$ & $120(66 \%)$ \\
\hline HBV & $13(7 \%)$ \\
\hline $\mathrm{HBV}+\mathrm{HCV}$ & $4(2 \%)$ \\
\hline Alcoholism & $24(13 \%)$ \\
\hline Unknown & $21(12 \%)$ \\
\hline MELD-Na, median (IQR) & $9.7(7.5$ to 13.3$)$ \\
\hline MELD-Na_90, median (IQR) & $11.8(8.5$ to 18.8$)$ \\
\hline
\end{tabular}

\section{Results}

3.1. Patient Characteristics. 182 HCC patients met all eligibility criteria and are included in the present analysis. Patient characteristics are summarized in Table 1 . The mean age was 62 , and $73 \%$ of patients were male. $54 \%$ of patients were Hispanic, and $66 \%$ had HCV infection as the sole etiology of liver disease. $66 \%$ underwent TACE and/or RFA, $39 \%$ received systemic therapy, and only $10 \%$ underwent resection. Median follow-up for all patients was 12.3 months. For surviving patients, median follow-up was 21.1 months.

3.2. MELD-Na and MELD-Na_90 Scores. The median baseline MELD-Na was 9.7 (IQR, 7.5-13.9), and median MELDNa_90 was 11.8 (IQR, 8.5-18.8). MELD-Na_90 was higher than MELD-Na for 113 patients (62\%), and the median percent change from MELD-Na to MELD-Na_90 was an increase of $9 \%$ (IQR, $-4 \%$ to $55 \%$ ).

3.3. Baseline Variables as Prognostic Factors. 110 deaths (60\%) were observed during the follow-up period. Median actuarial survival was 17.1 months, and 12 and 24-month actuarial survival rates were $61 \%$ and $39 \%$, respectively.

Univariate and multivariable Cox modeling results identifying predictors of OS among baseline variables, including

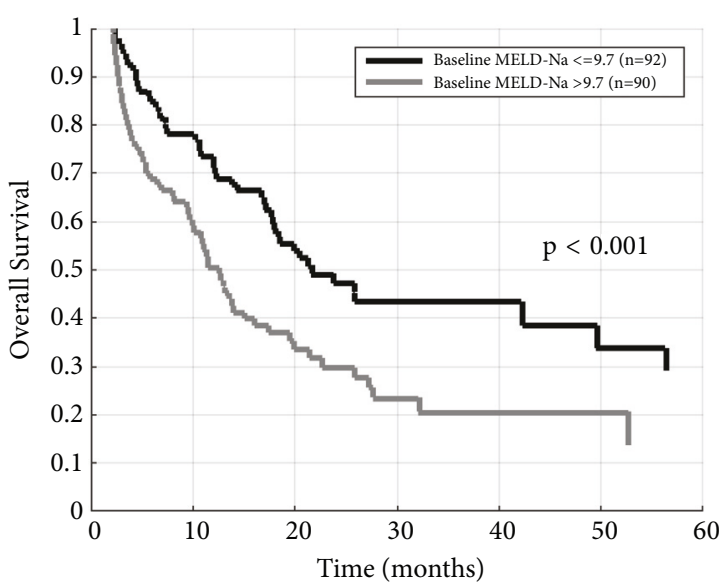

Figure 1: Kaplan-Meier overall survival curves after grouping patients by baseline MELD-Na. p value calculated using log-rank test.

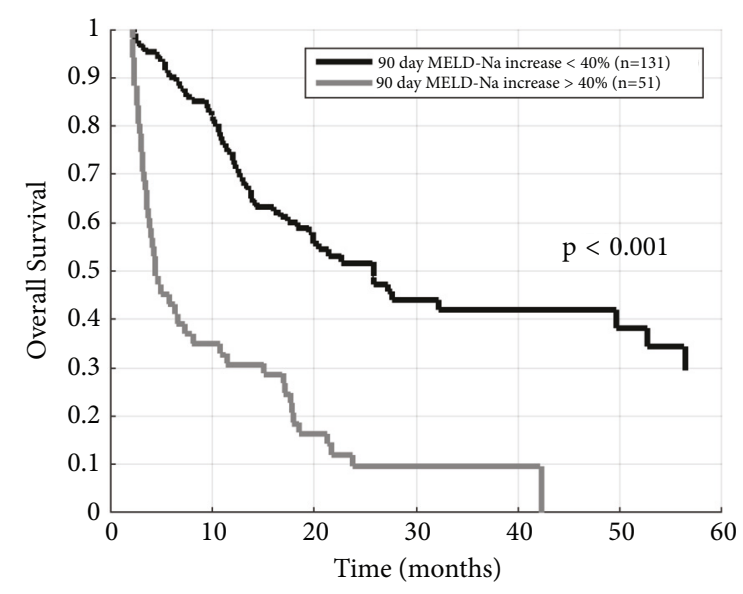

FIGURE 2: Kaplan-Meier overall survival curves after grouping patients using the optimal MELD-Na increase cutoff of $40 \%$. p value calculated using log-rank test.

MELD-Na, are displayed in Table 2. In the multivariable model, clinical stage $(\mathrm{HR}=2.66$ for stage III compared to stage I, 95\% CI 1.64 to $4.32, \mathrm{p}<0.001)$ and baseline MELD$\mathrm{Na}(\mathrm{HR}=1.05$ per unit increase, $95 \%$ CI 1.01 to $1.09, \mathrm{p}=0.012)$ were independently associated with increased mortality risk. Kaplan-Meier survival curves of patients by baseline MELD$\mathrm{Na}$ are shown in Figure 1.

3.4. Change in MELD-Na as a Prognostic Factor. The optimal prognostic cut-point for 90-day increase in MELD-Na was found to be $40 \% .51$ patients (28\%) demonstrated increases in MELD-Na exceeding 40\%. Median survival among patients whose MELD-Na increased by more than $40 \%$ was 4.5 months, and median survival among the 131 other patients was 25.8 months (log-rank p<0.001, Figure 2).

A multivariable Cox proportional hazards model including baseline MELD-Na and the change in MELD-Na at 90 days demonstrated that both baseline MELD-Na $(\mathrm{HR}=1.07$ per unit increase, $95 \%$ CI 1.03 to $1.11, \mathrm{p}<0.001$ ) and increase 


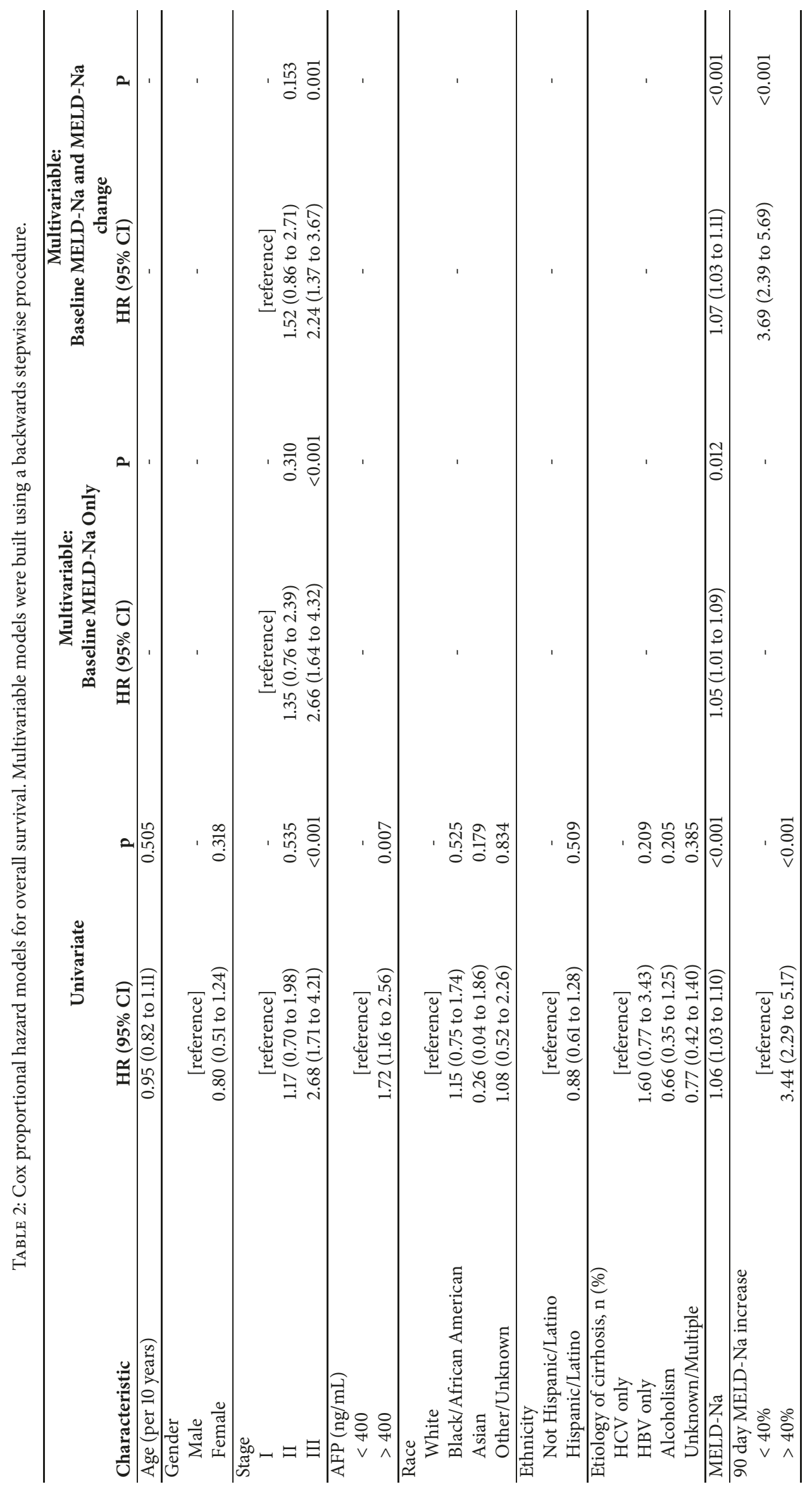


TABLE 3: Initial treatment modality grouped by MELD-Na increases cutoff of $40 \%$. One patient had missing information regarding initial treatment modality. TACE $=$ transcatheter arterial chemoembolization, RFA = radiofrequency ablation, Y-90 =yttrium-90 radioembolization, and SBRT = stereotactic body radiation therapy. Total percent may not add up to $100 \%$ due to rounding.

\begin{tabular}{|c|c|c|}
\hline \multirow{2}{*}{ Initial Treatment Modality Within 60 Days of Diagnosis } & \multicolumn{2}{|c|}{ MELD-Na Increase from Baseline } \\
\hline & $<40 \%, \mathrm{n}=131$ & $\geq 40 \%, \mathrm{n}=51$ \\
\hline TACE, n (\%) & $61(47)$ & $18(35)$ \\
\hline RFA, n (\%) & $18(14)$ & $5(10)$ \\
\hline Sorafenib, n (\%) & $14(11)$ & $9(18)$ \\
\hline Resection, n (\%) & $13(10)$ & $1(2)$ \\
\hline Y-90, n (\%) & $10(8)$ & $2(4)$ \\
\hline SBRT, n (\%) & $0(0)$ & $1(2)$ \\
\hline Transplant, n (\%) & $1(1)$ & $0(0)$ \\
\hline None, $\mathbf{n}(\%)$ & $13(10)$ & $15(29)$ \\
\hline
\end{tabular}

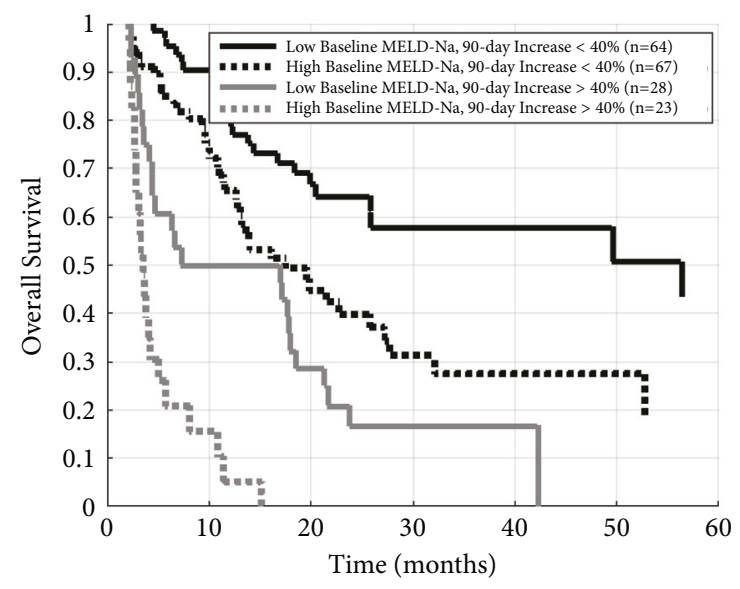

FIGURE 3: Kaplan-Meier overall survival curves after grouping patients by both baseline MELD-Na and MELD-Na increase. "Low" and "High" baseline MELD-Na groups defined as $\leq 9.7$ or $>9.7$, respectively.

in MELD-Na exceeding 40\% $(\mathrm{HR}=3.69,95 \%$ CI 2.39 to $5.69, \mathrm{p}<0.001)$ were independently associated with increased mortality risk. There was no evidence of interaction between these two measures as predictors of mortality (interaction term $\mathrm{p}=0.519)$.

Kaplan-Meier survival curves after dividing patients into four groups based on baseline MELD-Na and the change in MELD-Na are shown in Figure 3. Patients with baseline MELD-Na less than or equal to the median value of 9.7 whose MELD-Na increased by less than $40 \%$ at 90 days had the most favorable prognosis, with a median survival of 56.3 months. In contrast, median survival for patients with baseline MELD-Na exceeding 9.7 whose MELD-Na increased by more than $40 \%$ was only 3.6 months. As summarized in supplementary Table 1, these patients were more likely to die from both progression of their liver disease $(p<0.001)$ and from cancer-related death $(\mathrm{p}=0.013)$ than patients whose MELD-Na increased by less than $40 \%$ at 90 days. In addition, these patients were more likely to receive either systemic therapy $(18 \%$ versus $11 \%, \mathrm{p}<0.001)$ or no therapy $(29 \%$ versus $10 \%, \mathrm{p}<0.001)$ in comparison to the group with more favorable prognosis as shown in Table 3.

\section{Discussion}

The present study evaluated the utility of MELD-Na and 90day changes in MELD-Na scores as predictors of mortality in a predominantly $\mathrm{HCV}$-infected, racially diverse population of patients with nonmetastatic HCC. We demonstrated that both measures are powerful prognostic factors in this patient population. Used in combination, these measures may further define HCC patient subgroups with strikingly different prognoses.

The MELD-Na score was first shown to predict mortality in a predominantly Asian and HBV-infected HCC cohort $[11,17]$. This observation has not been replicated in a Western population, where HCC patients have a different demographic and etiologic profile. While both chronic HBV and HCV are risk factors for HCC, development of HCC in relation to stage of liver disease is somewhat different for both viruses, likely due to differences in carcinogenesis between HCV and HBV. We have demonstrated that MELD$\mathrm{Na}$ at the time of diagnosis is likely a relevant prognostic factor in our patient population that may be incorporated into management decisions and used to counsel patients.

After verifying the utility of MELD-Na as a prognostic factor in our cohort, we additionally demonstrated that an increase in MELD-Na score after 90 days exceeding $40 \%$ was associated with increased mortality risk. The dramatic increase in MELD-Na velocity in combination with high baseline MELD-Na > 9.7 identified a group of nonmetastatic HCC patients that have extremely poor prognosis with median survival of fewer than 4 months in our cohort. These patients have higher rates of mortality from both cancer-related death and from worsening of underlying liver disease. An increase in MELD-Na score denotes worsening hepatic function, as increase in MELD or MELD-Na score is correlated with hepatocyte loss $[5,18]$. Rapid increase in MELD-Na may be indicative of rapid disease progression impairing hepatocyte function and/or deterioration of liver function as a consequence of liver-directed therapy. This 
finding is evident when we evaluated the choice of initial treatment modality and cause of death within each group. The patients with rapid increase in MELD-Na are more likely to die from cancer-related death rather than from liver disease. Our findings are in agreement with other groups that had demonstrated patients with HCC can die from complications both due to their underlying liver disease as well as from cancer progression [19].

Previous reports have examined changes in MELD as predictors of overall survival in patients with cirrhosis. In one study, a rate of MELD increase exceeding 2.5 points per month was strongly associated with 6- and 12-month mortality [20]. For comparison, the $40 \%$ increase in MELD$\mathrm{Na}$ that we found to be the optimal prognostic factor in the current study corresponded to an increase of approximately 1 point per month for most patients.

Recalculated MELD scores after treatment with selective internal radiation therapy have previously been shown to be prognostic for overall survival [21]. The hazard ratio associated with a unit increase in MELD in that study was similar to the hazard ratio associated with a unit increase in MELD-Na in the present analysis. Additional variables that merit further exploration include volumetric tumor burden and initial response to therapy [21].

The current study has several limitations that must be acknowledged. Some missing data (e.g., cirrhosis etiology) could not be found in this retrospective study. Available data did not allow us to determine if 90 days following diagnosis is the optimal time at which to assess for changes in MELD$\mathrm{Na}$. Imaging data to allow assessment of disease progression were at times unavailable, precluding the inclusion of this important variable in our survival models.

In conclusion, our findings provide evidence for the general applicability of the MELD-Na score as a prognostic indicator for HCC patients, regardless of etiology. We have identified the velocity of change over 90-days in MELD$\mathrm{Na}$ score as a potentially important prognostic factor. Care must be taken in interpreting the data as many HCC patients on the liver transplant waiting list have low risk of dying, and overemphasizing the mortality risk may lead to further disparity in the liver allocation system $[22,23]$. However, our findings have identified a potential subgroup of HCC patients that have a significantly worse prognosis when compared to the majority of the HCC population that is not captured based on the traditional MELD-Na score [7]. Validation of these findings using additional datasets is warranted.

\author{
Abbreviations \\ HCC: Hepatocellular carcinoma \\ MELD: Model for end-stage liver disease \\ MELD: Na-Model for end-stage liver disease and sodium \\ HBV: Hepatitis B \\ HCV: Hepatitis C \\ INR: International normalized ratio \\ MMC: Montefiore Medical Center \\ PCR: Polymerase chain reaction \\ AFP: Alpha fetoprotein \\ TACE: Transcatheter arterial chemoembolization
}

RFA: Radiofrequency ablation

IQR: Interquartile range.

\section{Data Availability}

The patient data used to support the findings of this study are restricted by the Albert Einstein College of Medicine Institutional Review Board in order to protect patient privacy. Data are available for researchers who meet the criteria for access to confidential data.

\section{Conflicts of Interest}

The authors declare that they have no conflicts of interest.

\section{Acknowledgments}

This work was supported by the National Center for Advancing Translational Sciences (NCATS), a component of the National Institutes of Health (NIH), through CTSA Grants nos. UL1TR000086, TL1RR000087, and KL2TR000088. Its contents are solely the responsibility of the authors and do not necessarily represent the official views of the NIH.

\section{Supplementary Materials}

Table 1. Cause of death grouped by MELD-Na increase cutoff of $40 \%$. (Supplementary Materials)

\section{References}

[1] T. M. Welzel, B. I. Graubard, S. Quraishi et al., "Populationattributable fractions of risk factors for hepatocellular carcinoma in the United States," American Journal of Gastroenterology, vol. 108, no. 8, pp. 1314-1321, 2013.

[2] P. A. Farazi and R. A. DePinho, "Hepatocellular carcinoma pathogenesis: from genes to environment," Nature Reviews Cancer, vol. 6, no. 9, pp. 674-687, 2006.

[3] H. B. El-Serag, "Hepatocellular carcinoma," The New England Journal of Medicine, vol. 365, no. 12, pp. 1118-1127, 2011.

[4] P. Rougier, E. Mitry, J.-C. Barbare, and J. Taieb, "Hepatocellular Carcinoma (HCC): An Update," Seminars in Oncology, vol. 34, no. 1, pp. S12-S20, 2007.

[5] F. Botta, E. Giannini, P. Romagnoli et al., "MELD scoring system is useful for predicting prognosis in patients with liver cirrhosis and is correlated with residual liver function: a European study," Gut, vol. 52, no. 1, pp. 134-139, 2003.

[6] P. S. Kamath, R. H. Wiesner, M. Malinchoc et al., "A model to predict survival in patients with end-stage liver disease," Hepatology, vol. 33, no. 2, pp. 464-470, 2001.

[7] R. Wiesner, E. Edwards, R. Freeman et al., "Model for endstage liver disease (MELD) and allocation of donor livers," Gastroenterology, vol. 124, no. 1, pp. 91-96, 2003.

[8] A. S. M. Martins, H. Cortez-Pinto, P. Marques-Vidal et al., "Treatment and prognostic factors in patients with hepatocellular carcinoma," Liver International, vol. 26, no. 6, pp. 680-687, 2006.

[9] J. L. Limquiaco, G. L. H. Wong, V. W. S. Wong, P. B. S. Lai, and H. L. Y. Chan, "Evaluation of Model for End Stage Liver Disease (MELD)-based systems as prognostic index for hepatocellular 
carcinoma," Journal of Gastroenterology and Hepatology, vol. 24, no. 1, pp. 63-69, 2009.

[10] S. W. Biggins, W. R. Kim, N. A. Terrault et al., "Evidence-based incorporation of serum sodium concentration into MELD," Gastroenterology, vol. 130, no. 6, pp. 1652-1660, 2006.

[11] T.-I. Huo, H.-C. Lin, C.-Y. Hsia et al., "The MELD-Na is an independent short- and long-term prognostic predictor for hepatocellular carcinoma: A prospective survey," Digestive and Liver Disease, vol. 40, no. 11, pp. 882-889, 2008.

[12] C.-H. Chen, G.-T. Huang, P.-M. Yang et al., "Hepatitis Band C-related hepatocellular carcinomas yield different clinical features and prognosis," European Journal of Cancer, vol. 42, no. 15, pp. 2524-2529, 2006.

[13] Q. Li, H. Li, Y. Qin, P. P. Wang, and X. Hao, "Comparison of surgical outcomes for small hepatocellular carcinoma in patients with hepatitis B versus hepatitis C: A Chinese experience," Journal of Gastroenterology and Hepatology, vol. 22, no. 11, pp. 1936-1941, 2007.

[14] S. Roayaie, M. B. Haim, S. Emre et al., "Comparison of surgical outcomes for hepatocellular carcinoma in patients with hepatitis B versus hepatitis C: A western experience," Annals of Surgical Oncology, vol. 7, no. 10, pp. 764-770, 2000.

[15] E. Bellin, D. D. Fletcher, N. Geberer, S. Islam, and N. Srivastava, "Democratizing information creation from health care data for quality improvement, research, and education-The montefiore medical center experience," Academic Medicine: Journal of the Association of American Medical Colleges, vol. 85, no. 8, pp. 1362-1368, 2010.

[16] C. Liu, "Value of $\alpha$-fetoprotein in association with clinicopathological features of hepatocellular carcinoma," World Journal of Gastroenterology, vol. 19, no. 11, p. 1811, 2013.

[17] T.-I. Huo, C.-Y. Hsia, Y.-H. Huang et al., "Selecting a short-term prognostic model for hepatocellular carcinoma:comparison between the model for end-stage liver disease (MELD), MELDsodium, and five cancer staging systems," Journal of Clinical Gastroenterology, vol. 43, no. 8, pp. 773-781, 2009.

[18] A. Katoonizadeh, F. Nevens, C. Verslype, J. Pirenne, and T. Roskams, "Liver regeneration in acute severe liver impairment: a clinicopathological correlation study," Liver International, vol. 26, no. 10, pp. 1225-1233, 2006.

[19] O. F. M. Couto, I. Dvorchik, and B. I. Carr, "Causes of death in patients with unresectable hepatocellular carcinoma," Digestive Diseases and Sciences, vol. 52, no. 11, pp. 3285-3289, 2007.

[20] T.-I. Huo, J.-C. Wu, H.-C. Lin et al., "Evaluation of the increase in model for end-stage liver disease $(\triangle \mathrm{MELD})$ score over time as a prognostic predictor in patients with advanced cirrhosis: risk factor analysis and comparison with initial MELD and ChildTurcotte-Pugh score," Journal of Hepatology, vol. 42, no. 6, pp. 826-832, 2005.

[21] Z. Weng, J. Ertle, S. Zheng et al., "A new model to estimate prognosis in patients with hepatocellular carcinoma after yttrium-90 radioembolization," PLoS ONE, vol. 8, no. 12, p. e82225, 2013.

[22] D. Goldberg, B. French, P. Abt, S. Feng, and A. M. Cameron, "Increasing disparity in waitlist mortality rates with increased model for end-stage liver disease scores for candidates with hepatocellular carcinoma versus candidates without hepatocellular carcinoma," Liver Transplantation, vol. 18, no. 4, pp. 434-443, 2012.

[23] K. Washburn, E. Edwards, A. Harper, and R. Freeman, "Hepatocellular carcinoma patients are advantaged in the current liver transplant allocation system," American Journal of Transplantation, vol. 10, pp. 1643-1648, 2010. 


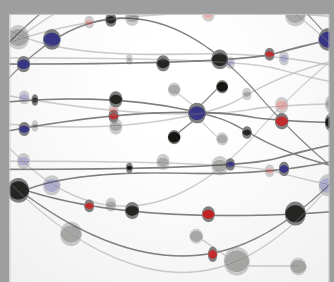

The Scientific World Journal
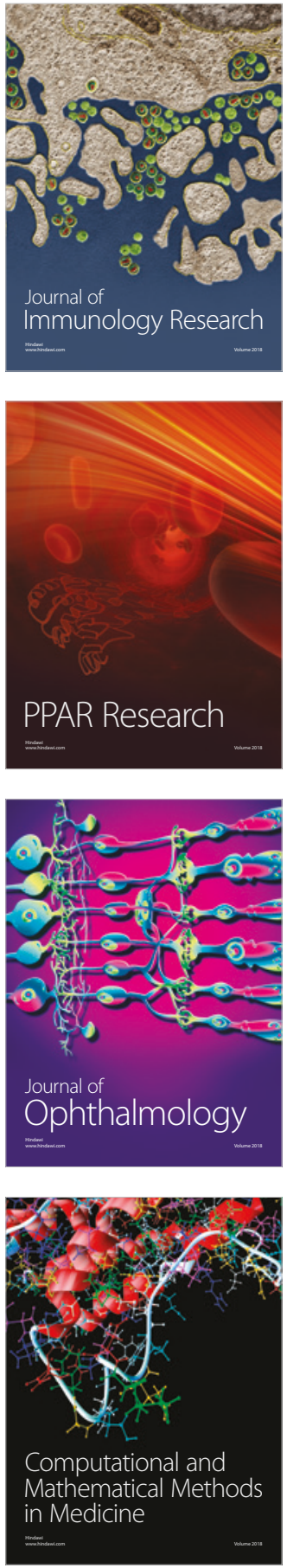

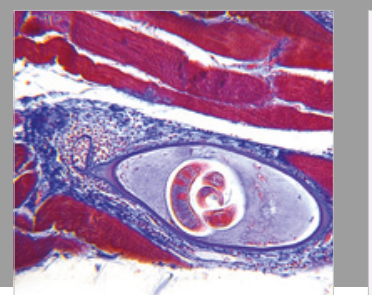

Gastroenterology Research and Practice

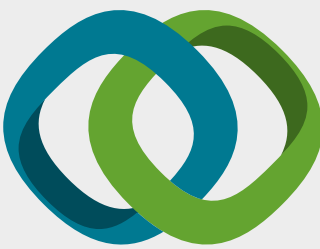

\section{Hindawi}

Submit your manuscripts at

www.hindawi.com
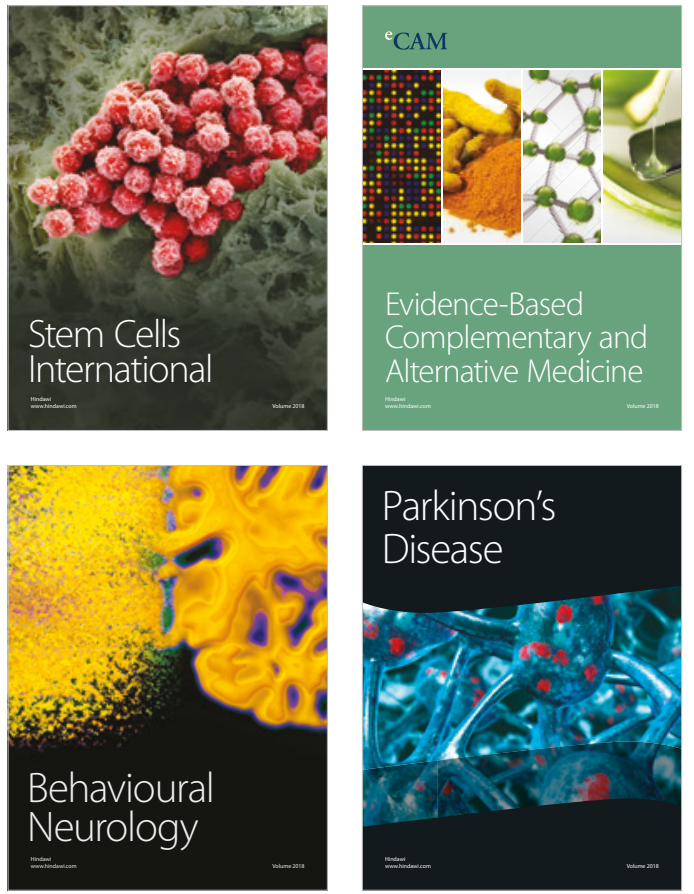

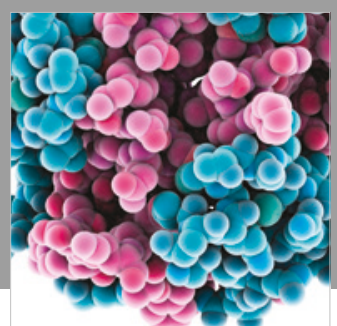

ournal of

Diabetes Research

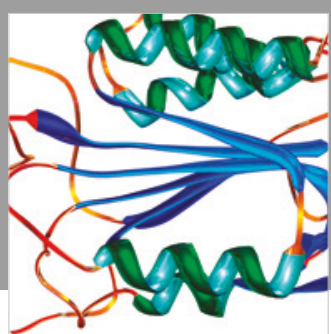

Disease Markers
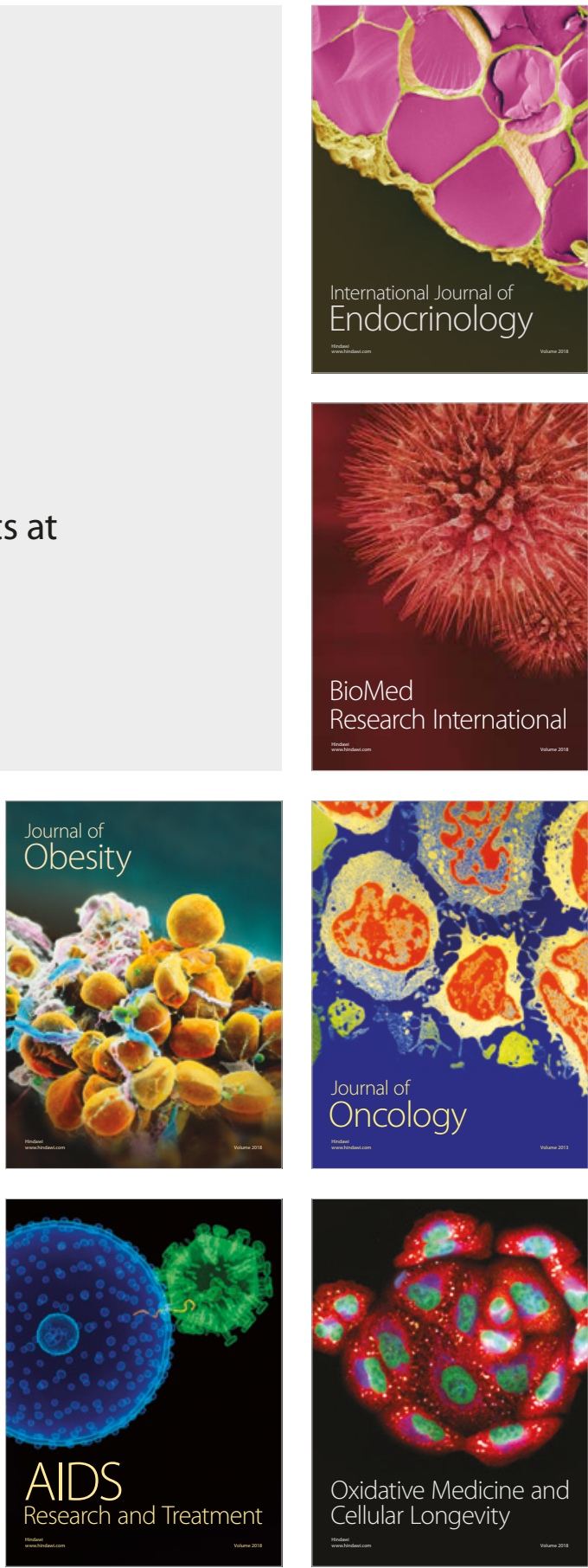